\title{
An Interactive Cardiac Tele Rehabilitation Program Using a Mobile Device
}

\author{
X Chen, CT Ho, ET Lim \\ Institute for Infocomm Research, Singapore, Singapore
}

\begin{abstract}
Patients recovering from a cardiac surgery need to undergo regular checkups and physical rehabilitation exercises at their local rehabilitation centers. It is timeconsuming and not personalized for each individual. Moreover, the stigma and emotional stress of re-visiting the rehabilitation center for some patients may prove to be more debilitating. With increasing pervasiveness of powerful mobile devices like pocket pc and Smartphone, this paper proposes a personalized mobile rehabilitation coaching program for mobile devices. It allows patients to perform their own cardiac fitness exercises and tests and forward their results to their physicians for assessment. Personalization in the rehabilitation exercises and test programs is achieved by the physicians by allowing direct communication with the patients and adjustments in the parameters via the testing program. A preliminary study has been conducted to evaluate the effectiveness of utilizing this personalized mobile rehabilitation program.
\end{abstract}

\section{Introduction}

With the increase in the amount of ubiquitous computing coupled with the improvement in mobile cellular technologies, frameworks are being devised to utilize this potential power. Health frameworks like MobiCare devised by Chen et al [1] utilizes such potential to improve the standard of living for people. As more people, especially the elderly, are afflicted by cardiovascular diseases, there is a rise in the need for cardiac treatment. Moreover, there is a proliferation of heart monitoring devices [2] in the commercial market. Chen et al [1] put together such devices with the use of a cellular phone to devise a framework, MobiCare to provide tele-monitoring. However, it can be extended to also provide telemedicine.

Given that people are becoming busier, telerehabilitation is becoming a more viable alternative to tradition rehabilitation. Traditionally, telerehabilitation began as the treatment of early stuttering with the use of plain old telephone service (POTS) Vaughn, G.R [3] and later with better POTS services saw a more collaborated framework to commit this form of treatment Werts et al [4]. However, given the increase in the numbers of the different kind of rehabilitation therapies and improvements in mobile technology, telerehabilitation can be extended to provide other forms of physical rehabilitation like brain rehabilitation after brain injury Brennan et al [5] or physical therapy to treat carpal tunnel syndrome [6].

This paper proposes the combination of the telerehabilitation with the MobiCare framework to provide a cardiac telerehabilitation system to patients who suffer from cardiac vascular diseases or have undergone heart surgeries. The motivation is for people who might have problems with travelling to a cardiac rehabilitation centre. Problems related could be, but not restricted to, travelling time, disability to travel or even psychological stigma of the morbidity of a cardiac rehabilitation centre. In a society where family ties are utmost importance, the stigma of uncertain death motivates more for cardiac patients to spend time with their families. Hence, a cardiac telerehabilitation system would be a viable medical alternative.

In section 2, a detail explanation of the cardiac telerehabilitation system is explained followed by the technical methodologies. Section 3 covers the experiment's methodologies and initial test setup. The paper will then conclude in section 4 with the results and a look on the future for such mobile applications.

\section{Cardiac rehabilitation exercises with senses}

The Cardiac Rehabilitation Exercises with Senses (CREWS) can be separated into 2 phases. Phase 1 involves the appropriate cardiac exercise regime that is to be followed and executed by the patient. Phase 2 involves the usage of the evaluation program on the cardiac health of the patient after each regime of exercises.

This would achieve the close equivalent settings of the settings a patient undergoing cardiac rehabilitation. CREWS is providing an augmentation to conventional cardiac rehabilitation and is not seeking to replace it. 
Hence, CREWS seeks to augment the current processes. The processes and flow on the cardio rehabilitation system are shown in Figure 1.

\section{Cardio Telerehabilitation System}

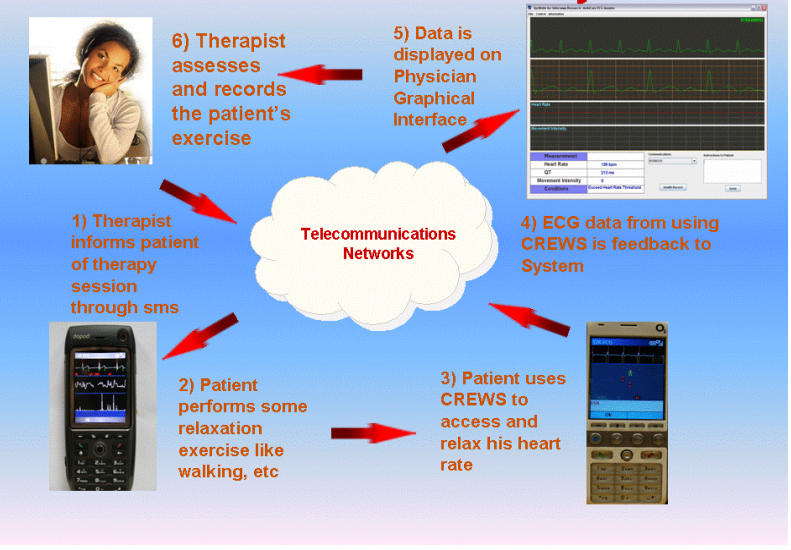

Figure 1: Processes and Flow

\subsection{Phase 1: cardiac rehabilitation exercise}

In phase 1 , Chen et al [1] initial MobiCare program is used as a facilitator to initial the cardiac rehabilitation program. The therapist would initiate the program by sending a text message to the patient via his mobile phone. Upon receiving such a text message, the user would then proceed with the exercises using the MobiCare program to monitor his Electro-cardiogram (ECG) and movement activities.

As an added bonus, the MobiCare program can also double as detector for any abnormal activity that might occur during the course of the exercise. These activities can be arrhythmias or abnormal correspondence of ECG and movement activities.

\subsection{Phase 2: cardiac evaluation with senses}

Phase 2 occurs after the exercises in Phase 1. This is the phase to test if the patient has the ability to relax and calm his heart down with CREWS.

CREWS targets to calm the patient down by bringing down his heart rate. Like a game, CREWS has several stages that increase with difficulty progressively. The increase in difficulty is the actual progressive lowering of the heart rate threshold for the patient to achieve. At each stage, there is a target heart rate threshold to achieve and maintain. Progression to the next stage occurs only when the threshold is achieved and maintained over a period of time. Each progression to the next stage is transparent to the patient. And this is done in incremental stages to ensure that the patient does not stress his heart even more as the sudden fluctuation of heart rate my proved to be even more fatal.

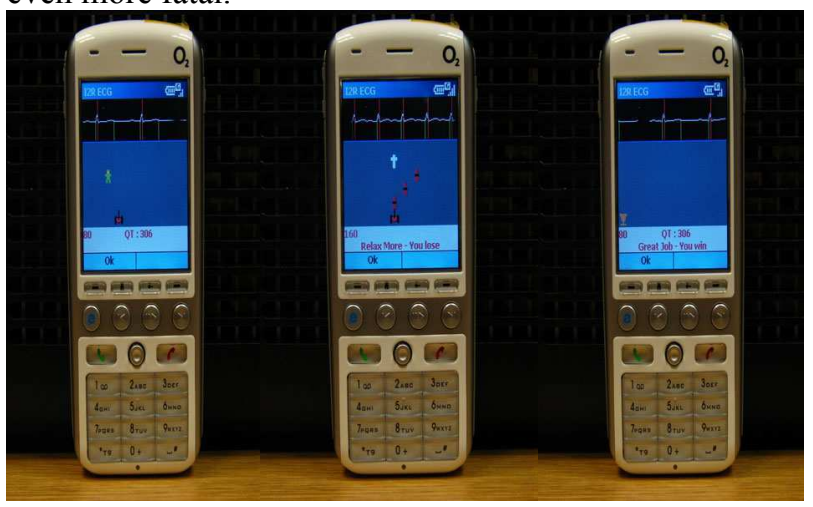

Figure 2: CREWS Rehabilitation Program

CREWS a simple 2-D game where the patient is a spy set to cross the enemy lines without alerting the enemies. The spy will have to move from left to right for a period of time without alerting the enemy. If the spy can maintain his presence at that stage long enough, he will progress onto the next stage where the enemy is more easily alerted due to the closer in proximity between the spy and the enemy. The enemy is a red colored tank that is set to fire missiles at the spy whenever the patient's heart rate crosses a pre-defined threshold; heart rate crossing the threshold is the alert that will prompt the enemy to shoot missiles at the spy. If the missiles are fired, the spy will try to move to dodge it.

While the game is in progress, ECG data is also simultaneously being transmitted back to the cardiac rehabilitation centre. The progress of the exercise can then be monitored and checked by the therapist at the cardiac rehabilitation center. The therapist can then assess the patient's cardiac well-being and decide if the level of difficulty of the exercise should be increased. The setting of the difficulty can be adjusted using the "Instructions to Patients" text box on the physician user interface while still maintaining its original functions [1].

\subsection{Cardiac rehabilitation program}

By combining phase 1 and 2 together, we experimented on a small group of people to see what outcome would come out of the cardiac rehabilitation program. The participants were required to follow a strict regime to ensure that the later comparisons of the results would not be skewed.

The experiments were conducted on a daily basis close to the end of the day for 1 week per participants. Before the experiment commences, each participants were required calm down to stabilize their heart rate for a minute. Then they were asked to commence their rehabilitation exercise of walking on the treadmill for 10 
minutes. They will walk according to the speed and time duration given in the graph below.

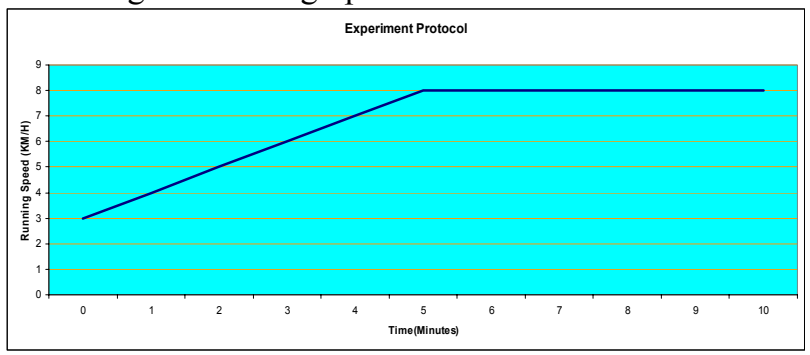

Figure 3: Exercise Intensity Chart Over Time

After the whole 10 minutes, they would switch to phase 2 to calm themselves using CREWS. This would exercise their ability to calm themselves down and to achieve control of their heart rate.

\section{Results}

For the experiments, the ECG of each participant of the experiment was recorded onto the memory card in the smartphone. The ECG is trend converted into instant rate so that a heart rate trend graph could be plotted. Below is diagram of the heart rate trend.

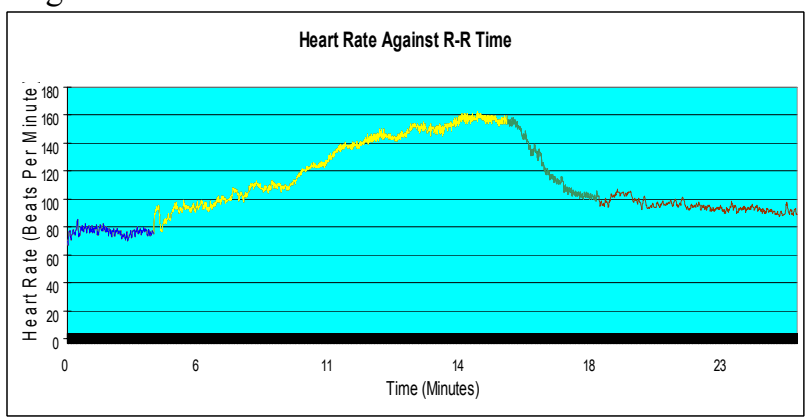

Figure 4: Heart Rate Trend During the Experiment

These ECG readings would provide an insight to the heart rate trends of each individual participant as they were taking the experiments. As mentioned earlier, the different colour bands in Figure 3 corresponds to different stages of the experiment. The blue band is the stabilizing of the heart rate stage. The yellow band is the exercise stage of doing the 10 minutes incremental walking. The green band is the stage where the participant doing the exercise uses CREWS to reduce his heart rate. And the final brown band is the recovery to normal period. Hence, this is the typical trend that most participants should experience.

The focus of the experiment was to determine if the overall cardio-rehabilitation system was able to help users achieve better heart rate control. Therefore, the yellow and green band of the heart rate trend graph is of importance. For the yellow band, a linear progression of the heart rate should be observed. This would be indicative that the heart rate is able to adapt to stress steadily. For the green band, an exponential decrease of the heart rate should be observed. This would be indicative of the heart flexibility to slow down itself once the physical exertion is over and that the participant is able to achieve some control of his ability to calm his heart down after his body has went through some physical exertion.

And also the heart rate should reduce by at least 35 beats per minute after the whole exercise as an indicator of reduced chances of sudden death via a heart attack. [7]

After conducting the experiments on some participants of age 25-45 over the period of 5 working days, the following data were obtained.

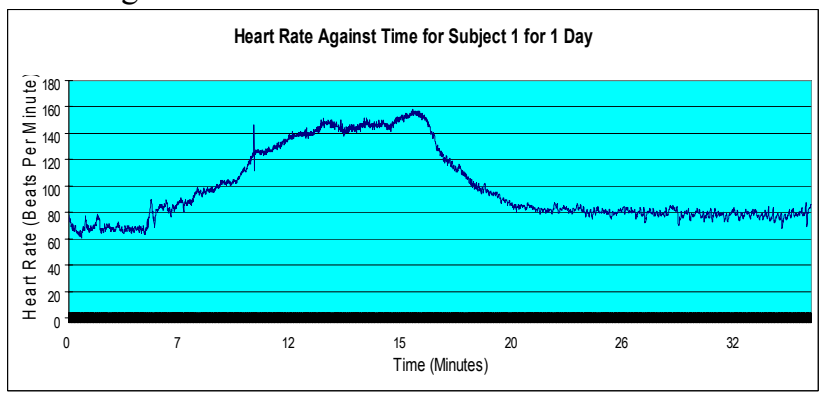

Figure 5: Day 1 - Subject 1

The above is the heart rate of subject 1 after completing the whole experiment for the first time. Below is the heart rate of subject 2 after completing the experiment on day 1 .

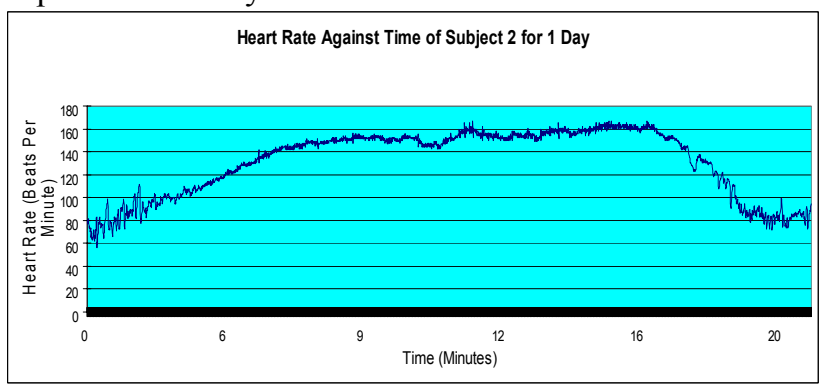

Figure 6: Day 1 - Subject 2

We see 2 very different individuals. It is observed that both the heart rate acceleration and deceleration are very different. The specific trend that we are looking for was not existing in both subject's heart rate readings.

We have collected and plotted the heart rate trend of subject 1 and subject 2 for the duration of 1 week. We do see the changes in the shape of the graphs as each day goes by. Discounting the initial and ending noise due to starting up and taking down of the apparatus, it was observed that the acceleration of the heart rate is achieving a more linear pattern and the deceleration a 
more exponential pattern towards the end of the week. And it was also interesting to note that the heart rate after exercise could not revert back to the original resting heart rate before the exercise.

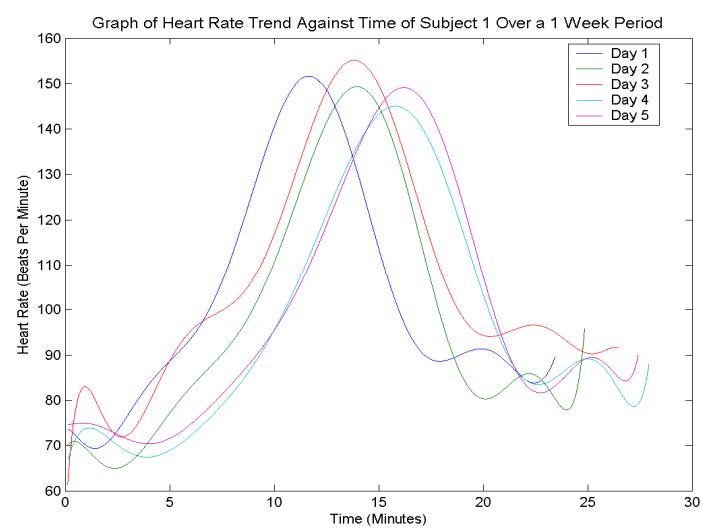

Figure 7: Heart Rate Trends - Subject 1

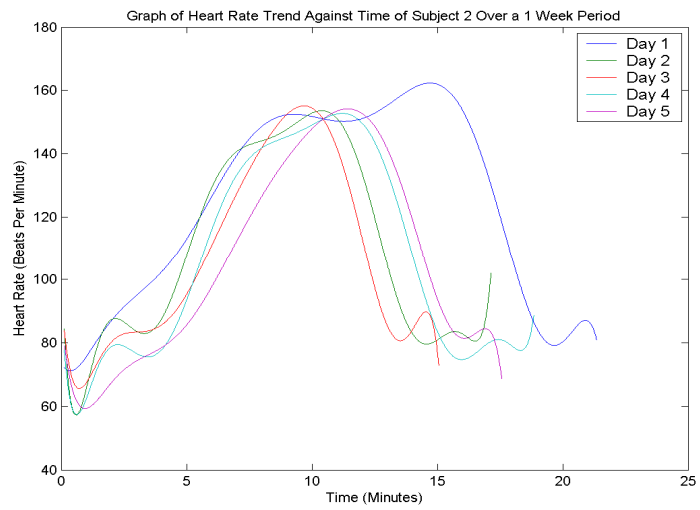

Figure 8: Heart Rate Trends - Subject 2

After one month, the heart rate trend the test subject was obtained as shown in the graph below.

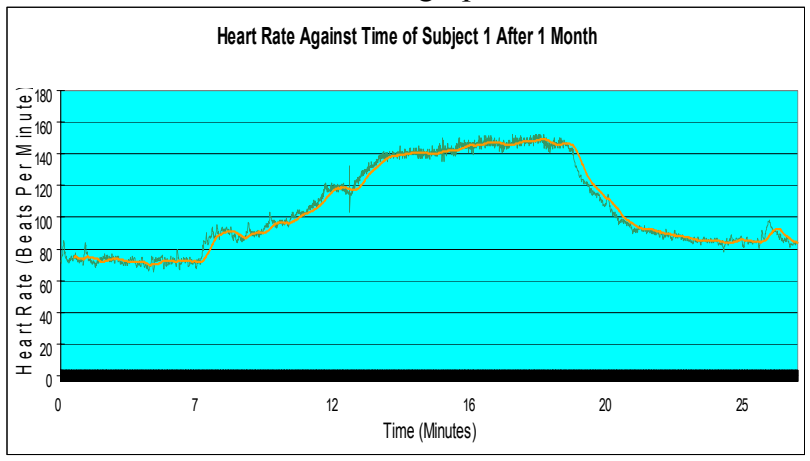

Figure 8: Experiment After 1 Month

The hypothesis that we first set out in the experiment was we needed a heart rate trend that showed a gradual increase in the heart rate during the phase one period and an exponential decrease in the heart in the phase two period. This was observed in the heart rate trend of figure 8. A sign that was most-promising for this experiment.

\section{Conclusions}

Although the results were beginning to show just after five days into the experiment, more experiments were needed to attest to the trend that we first set out to obtain. Hence, one month after it all began we were able to see that trend that was required. This is a very preliminary trial on its own, more experiments and collaborations with clinicians are required to derive more conclusive results. We are working with local hospitals on MobiCare and we hope to introduce CREWS to them for trials.

It is demonstrated in this paper that the Cardiac Telerehabilitation System is a viable alternative for patients that are on cardiac rehabilitation. It is able to allow people to adhere to their program no matter the time of the day and location. It also removed from them the social stigma of going to a rehabilitation centre. Plus, it still has similar care and concern that they will be getting if they visited a local cardiac rehabilitation centre. This system can be part of a more holistic healthcare system that tends to the overall health of an individual.

\section{References}

[1] X Chen, CT Ho, ET Lim, TZ Kyaw. Cell Phone Based Online ECG Processing for Ambulatory and Continuous Detection. Computers in Cardiology 2007;34:653 - 6.

[2] Alive Heart Monitors, Alive Technologies Pty Ltd

[3] Vaughn GR. Tel-communicology: Healthcare Delivery Systems for Persons with Communicative Disorders. American Speech-Language-Hearing Association 1976;137.

[4] Wertz RT, Dronkers NF, Berstein-Ellis E, Shubitowski Y, Elman R, Shenaut GK, Knight RT. Appraisal and Diagnosis of Neurogenic Communication Disorders in Remote Settings. Clinical Aphasiology 1978;17;117-123

[5] Brennan DM, Georgeadis AC, Baron CR, Barker LM. The Effects of Videoconferencing-based Telerehabilitation on Story Retelling Performance by Brain-Injured Subjects and its Implications for Remote Speech-Language Therapy. Telemedicine Journal and E-Health 2004; 10;147-154

[6] Kuttuva M, Boian R, Merians A, Burdea G, Bouzit M, Lewis J. The Rutgers Arm, A Rehabilitation System in Virtual Reality: A Pilot Study. CyberPsychology \& Behavior 2006;9(2);148-152

[7] Jouven X, Empana JP, Schwartz P, Desnos M, Courbon D, Ducimetiere P. Heart-Rate Profile during Exercise as a Predictor of Sudden Death. The New England Journal of Medicine 2005; 352; 1951-58

Address for correspondence

Xiang, Chen

1 Fusionopolis Way, \#21-01 Connexis, Singapore 138632 xchen@i2r.a-star.edu.sg 